\title{
Dental attitudes and behaviours in 1998 and implications for the future
}

\author{
G. Bradnock, ' D. A. White, ${ }^{2}$ N. M. Nuttall, ${ }^{3}$ A. J. Morris, ${ }^{4}$ E. T. Treasure, ${ }^{5}$ and C. M. Pine, ${ }^{6}$
}

\author{
The 1998 Adult Dental Health Survey included face to face \\ interviews with participants to determine their dental attitudes \\ and behaviours. This article considers reported oral hygiene \\ practices, treatment choices, satisfaction with appearance of \\ teeth, attitudes towards wearing dentures and how these have \\ changed since previous surveys. Although overall there has \\ been a steady improvement in dental health attitudes, adults \\ from disadvantaged households are still lagging behind. This has \\ implications for social equity.
}

\begin{abstract}
A face-to-face interview, conducted in the Ahome by ONS interviewers, has always formed the backbone of the decennial studies into adult dental health. Over the years questions have been asked concerning what choices people would make about their oral health should they be faced with making a decision. Some questions relate to choices around visiting the dentist and others are about the choices of treatment available to them. Attitudes related to reported dental attendance are examined in a companion paper in this series. ${ }^{1}$ This paper looks at the reported oral hygiene practices of respondents, what choices they said that they would make in certain circumstances, their satisfaction with the appearance of their teeth, their attitudes towards the possibility of wearing dentures in the future and individual concerns expressed.

When the first adult dental health study of England and Wales was undertaken in 1968, $37 \%$ of adults were reported as edentate. In 1998 that appalling statistic has reduced to 12\% in England and Wales and 13\% in the UK. A further analysis of tooth loss can be found in a companion paper in this series. ${ }^{2}$ The dramatic changes in oral health over the last 40 years are partly the result of changes in
\end{abstract}

${ }^{1}$ Senior Lecturer in Dental Public Health, ${ }^{2}$ Lecturer in Oral Health, ${ }^{4}$ Lecturer in Dental Public Health, School of Dentistry, The University of Birmingham; ${ }^{3}$ Senior Research Fellow, ${ }^{6}$ Reader in Dental Public Health, Head of Section of Dental Public Health o Health Psychology, Dundee Dental School; ${ }^{5}$ Professor of Dental Public Health, University of Wales College of Medicine

e-mail:g.marchment@bham.ac.uk

REFEREED PAPER

Received 13.06.00; Accepted 31.07.00

(C) British Dental Journal 2001; 190: 228-232 attitude towards self-care and dental treatment. However, these same changes are also a factor in changing expectations and attitudes.

A series of questions were asked in the 1998 interview to investigate oral health behaviour and attitudes (Fig. 1). The 1998 responses to these questions are related to previous decades to establish whether attitudes have changed, with a view to determining the implications for the future of dental care. The basic oral health messages are now well embedded, in some form or

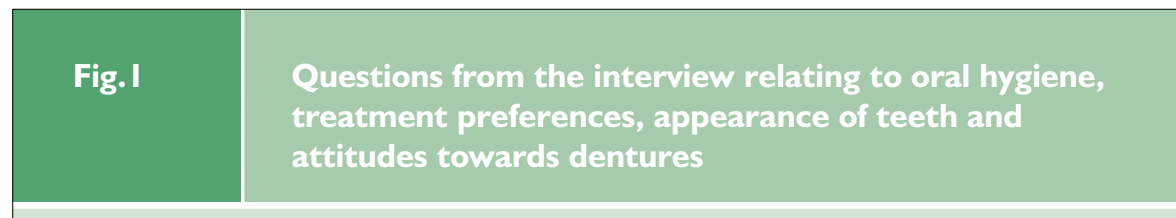

Oral hygiene

Do you usually keep your top/bottom plate in at night?

How often do you clean your teeth nowadays?

Do you use anything other than an ordinary toothbrush and toothpaste for dental hygiene purposes? If yes, what do you use?

Has a dentist or any of the dental staff demonstrated to you how to clean your teeth?

Treatment preferences

If you went to the dentist with an aching back tooth, would you prefer the dentist to take it out or fill it, supposing it could be filled? If the dentist said a front/back tooth would have to be extracted (taken out) or crowned, what would you prefer?

\section{Appearance}

In general, how do you feel about the appearance of your teeth (and/or dentures), are you satisfied or not satisfied with the way they look? If not, what is it about the way your teeth or dentures look that makes you not satisfied?

Dentures

Do you find the thought of losing all your own teeth and having full dentures very upsetting, a little upsetting or not at all upsetting? Do you find the thought of having a partial denture to replace some of your teeth very upsetting, a little upsetting or not at all upsetting?

NB This is a digest of questions asked in the survey.

For full details see reference 3 . 
another, in the folklore of the UK community. Phrases such as 'if you don't look after your teeth, you will lose them' are well known. Thus if people have some concerns about their future dental progress this may be reflected in the oral hygiene practices they claim to adopt.

\section{What oral hygiene behaviour do people report?}

A growing emphasis toward aesthetic considerations both in the media and dental practice is being demonstrated, therefore questions related to satisfaction with appearance can be considered as one barometer of oral health. Reported oral hygiene practices also reflect the level to which the UK population is receiving oral hygiene advice. Of those dentate adults questioned, $74 \%$ reported cleaning their teeth at least once a day. This comprised $83 \%$ of women and $64 \%$ of men. Figure 2 shows how this reported behaviour has changed since 1978. Amongst adults who claim to only attend the dentist when they have trouble, the proportion indicating that they brush at least twice a day has increased from $49 \%$ in 1978 to $61 \%$ in 1998 . This is a rise of $12 \%$ compared with only a $2 \%$ rise amongst reported regular attenders.

Amongst those who wore dentures, over half reported wearing their denture at night, in conflict with current dental advice.

Figure 3 shows changes in the reported use of oral hygiene products since 1978 . Whereas in 1978 almost $80 \%$ claimed to only use a toothbrush and toothpaste, 20 years later we can demonstrate that over $20 \%$ now indicate use of floss and/or a mouthwash in addition to toothbrushing. There was a clear indication that those from non-manual backgrounds were more likely than those from manual backgrounds to report using such additional methods. As these data are essentially interview responses, actual usage can only be estimated. It is possible that those from the non-manual backgrounds are more aware of alternative forms of oral hygiene and are probably more able to purchase floss and mouthrinses than those in lower income brackets. However the increased attention given to extra oral hygiene aids such as floss and mouthrinses in both professional and commercial campaigns at least makes people aware of what they should be using. It is interesting to note however that $38 \%$ of respondents cannot recall having been given tooth cleaning instruction or advice about gum care from their dentist.

There is clearly greater interest in using personal oral health products than was demonstrated in previous decades and these
Fig. 2 Frequency of tooth cleaning in the UK, 1978-1998

\begin{tabular}{l}
\hline \\
\hline Fig. 3 Use of oral \\
hygiene products \\
in the UK, \\
1978-1998 \\
\hline
\end{tabular}

improving trends appear to be stronger than those apparent for reported dental attendance. There appears to be very little evidence to assume that the public, even those who admit only attending the dentist when

\section{In brief}

- Attitudes towards dental health are more positive than in previous surveys of adult dental health

- Basic oral health messages are now well embedded in the folklore of the UK community

- Seventy-nine per cent of adults said they would rather have an aching back tooth filled than extracted

- Sixty-one per cent of adults with no denture experience were very upset at the thought of wearing full dentures in trouble, are apathetic about their dental health and their dental progress.

\section{What treatment might people prefer for their teeth?}

Since the inception of the studies, respondents have been asked whether they would prefer their front or back teeth to be extracted or restored and positive trends toward restoration have been observed over the decades. Responses to treatment preferences for front teeth could be regarded as having both oral health and aesthetic considerations, since extraction of front teeth imply adverse consequences for appearance. Conversely treatment of back teeth are less likely to be subject to these considerations and changes in attitude toward the treatment of posterior teeth might be considered as relating more closely to more positive attitudes to oral health. 


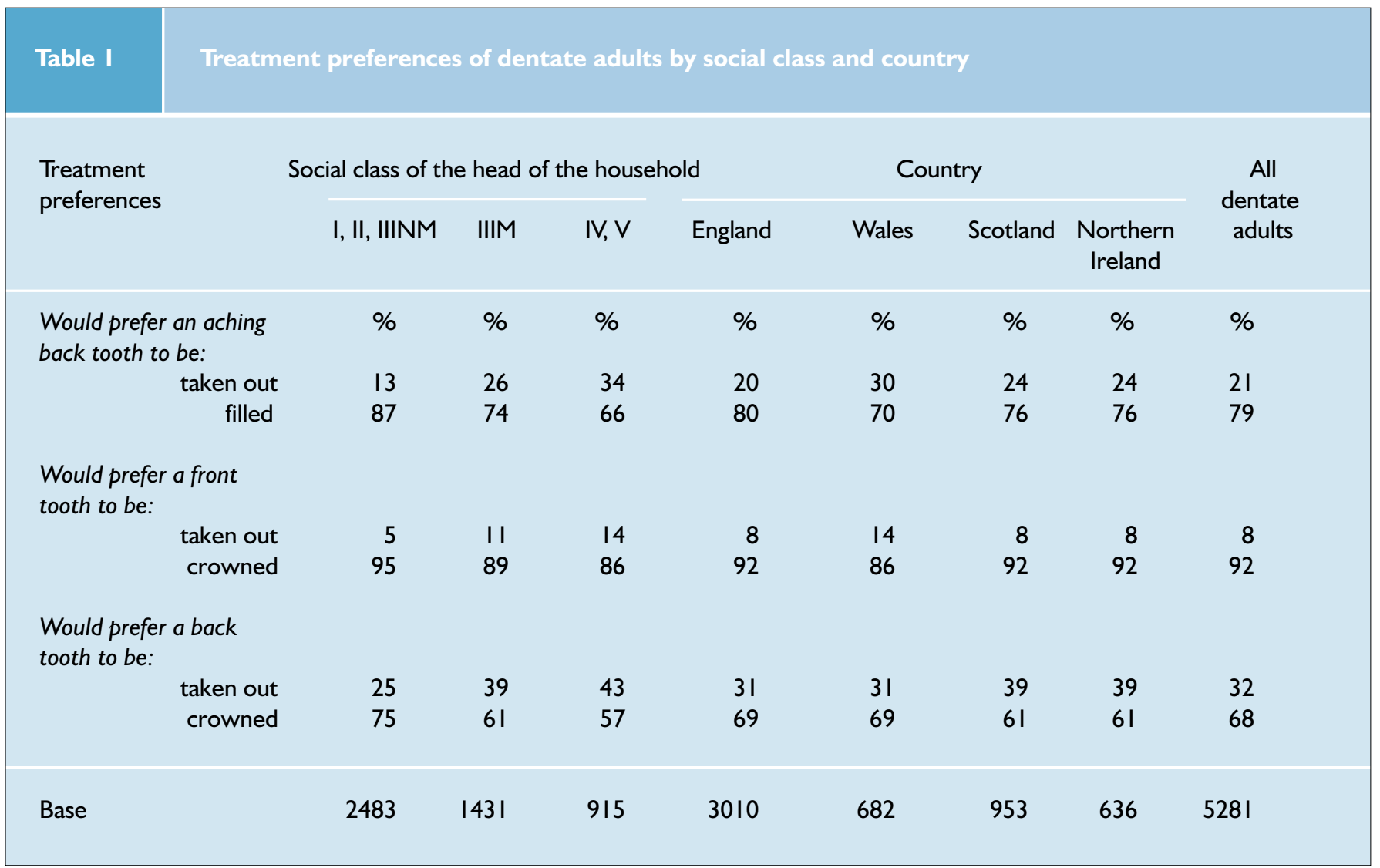

The questions that were asked relating to treatment preferences give an indication of attitudes towards retention of natural teeth. For front teeth, unsurprisingly, over $90 \%$ of adults said that they would prefer a crown to avoid an extraction, as this would almost inevitably lead to wearing of a denture for aesthetic considerations.

For back teeth just over three-quarters of adults overall $(79 \%)$ said they would rather have an aching back tooth filled rather than extracted. Although this represented only a small increase since the 1988 survey, it was a $15 \%$ increase since 1978 . When given the choice of a crown rather than an extraction, $68 \%$ of adults said they would prefer a crown.

Clear differences were apparent between adults from different social classes; those from non-manual backgrounds were more likely to prefer a restorative option for a tooth compared with adults from manual and unskilled backgrounds. For example, three-quarters of adults from non-manual backgrounds would rather have an aching back tooth crowned rather than extracted compared with $61 \%$ of those from manual and $57 \%$ of those from unskilled backgrounds (Table 1).

There were also differences between adults from different countries; those from England were least likely to prefer an extraction rather than a filling for an aching back tooth whereas adults from Wales were most likely to prefer an extraction. Those from Northern Ireland and Scotland were the most likely to prefer extraction to a crown for a back tooth.

Despite the fact that there are still some individuals who would prefer extractions to restorative treatment, clearly this is now a minority view and this again indicates a growing awareness of the UK population to the possibility of retaining their teeth for life.

\section{How satisfied are people with the appearance of their teeth?}

Aesthetic considerations can be a major oral health incentive to adults, particularly now that people are less likely to suffer high levels of disease and discomfort. With this in mind, questions relating to how satisfied people feel with their teeth have been included in the adult studies. Twenty-seven per cent of dentate adults indicated that they were dissatisfied with the appearance of their teeth, a similar proportion to 1988 , (Fig. 4). Thirty-six per cent of those who indicated that they only attended when in trouble were dissatisfied with the appearance of their teeth compared with only $23 \%$ of those who attended regularly. It would appear that $36 \%$ either do not feel strongly enough about this situation to consider it worth a visit to the dentist or they do not believe that the dentist can improve their appearance.

Table 2 shows the reasons given for dissatisfaction with dental appearance. The most common reason given was colour, followed by tooth alignment, spaces and broken teeth. 
Fig. 4

Dissatisfaction

with appearance

of teeth or

dentures in the

UK, 1988-1998
Percentage of

dentate adults (\%)

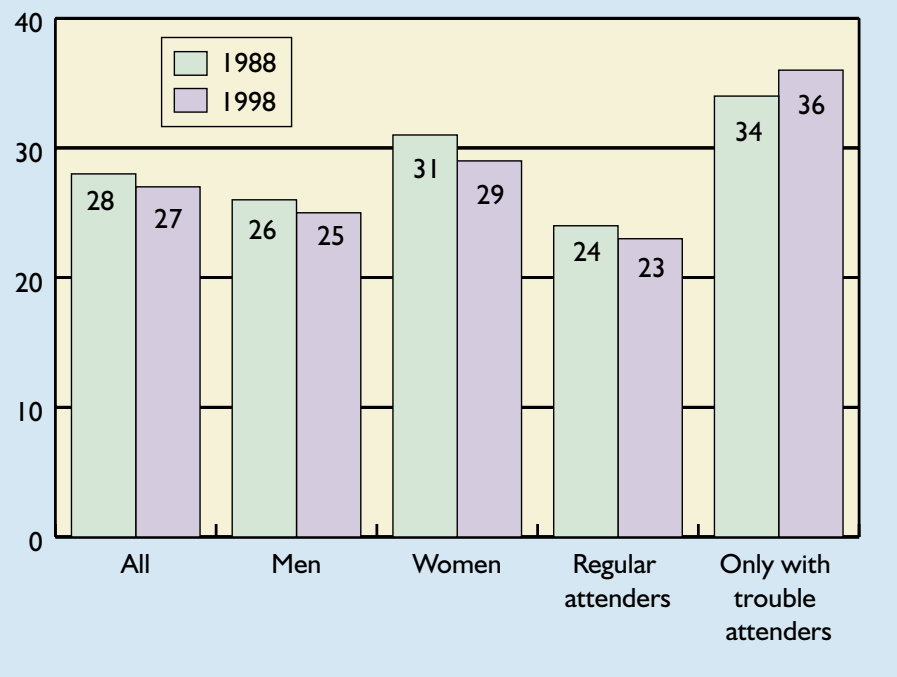

\section{What unsolicited opinions did people} offer?

Thirty-one per cent $(1,797)$ of adults participating in the interview responded to the opportunity to add further comments. Those who responded tended to be from the middle age-bands, from England and Wales, particularly the south of England and from the higher socio-economic groups. Although the comments varied widely certain themes and trends could be captured, showing an emphasis on cost concerns, a perceived drift of primary dental care away from the NHS and difficulty in accessing an NHS dentist. It remains unclear from these data whether these problems exist more among the groups outlined or whether these particular groups are more likely to add unsolicited comments.

\section{How do people feel about having dentures?}

Most adults still perceived that denture wearing was a possibility for them in the future. We have seen throughout the series of reports that adults increasingly wish to keep their own teeth and would be upset if they were to require dentures. In 1998, 28\% of adults were seen to still be reliant on complete or partial dentures. Since 1968, the perception of having to wear complete dentures as an inevitable feature of ageing has changed. It is now more likely for adults to consider the wearing of full dentures as stigmatic. In $1998,61 \%$ of adults who had no experience of wearing dentures were very upset at the thought of wearing complete dentures. However, only $27 \%$ were very upset at the thought of wearing partial dentures. These proportions did not alter dramatically when compared by reported regularity of attendance. A slightly greater proportion of those who reported regular attendance indicated that they would be very upset at having to wear full dentures (30\%) when compared with those who only attended when in trouble $(21 \%)$. This difference was not statistically significant however and cannot be confirmed to be the main reason why people regularly attend the dentist.

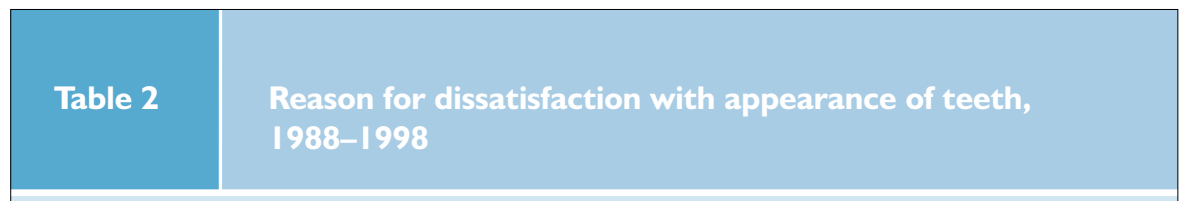

Dentate adults dissatisfied with appearance of teeth (or dentures)

United Kingdom

Reason for dissatisfaction 1988 1998

Colour of teeth

$\% \quad \%$

Crooked/slanting/protruding/irregular teeth

38

36

Gaps/spaces in mouth

13

Fillings/colour of fillings

II

Decayed teeth

Size/shape of teeth

Broken/chipped teeth

Need filling

Other

\section{$\%$}

48

34

18

10

$7 \quad 5$

69

$5 \quad 11$

03

I 8

Base

956

1416

Percentages may add to more than $100 \%$ as respondents could give more than one answer.

Base for 1988 as presented in the 1998 report 


\section{Implications for the future}

The data presented in the adult dental health surveys, over three decades of reporting, indicate a steadily improving approach toward more positive dental health attitudes. In particular there is a strong indication that adults increasingly wish to retain their natural teeth and are prepared to undertake certain procedures that have been recommended to them by the dental profession and others. The most important indicator must be the increase in people who would prefer their aching back tooth to be restored rather than extracted. These data are strengthened by the growing reported increase in toothbrushing and use of dental floss and mouthrinses.

There is an underlying concern apparent in the data demonstrating that those who have greater oral health needs and those from the more deprived households are still lag- ging behind in terms of their oral health attitudes. This is not surprising, but it continues to disappoint those who have worked toward equity in oral health. Increased use of oral health aids may be related to advice from the dentist, to advertisements in glossy magazines or to availability of oral health materials in the higher priced supermarkets and high street pharmacies. It is most likely to be a combination of these factors and it remains a major issue that one group of the population are not experiencing similar benefits to the others. It might be concluded that adult oral health continues to be a measure of social exclusion and as such this must be remedied.

This article has been refereed under the British Dental Journal reviewing process. Full details of sample numbers can be found in the survey report. We would like to acknowledge the work ofAlison Walker, Maureen Kelly and other staff of the Office for National Statistics. This work was undertaken by a consortium comprising the Office for National Statistics and the Dental Schools of the Universities of Birmingham, Dundee, Newcastle and Wales who received funding from the United Kingdom Health Departments; the views expressed in this publication are those of the authors and not necessarily those of the Health Departments nor of the other members of the consortium.

1 Nuttall N M, Bradnock G, White D A, Morris J, Nunn J H. Dental attendance in 1998 and implications for the future. Br Dent J 2001; 190: 122-127.

2 Steele J S, Treasure E T, Pitts N B,Morris A J, Bradnock G. Total tooth loss in the United kingdom in 1998 and implications for the future. Br Dent J 2000; 189: 598-603.

3 Kelly M, Steele J, Nuttall N, Bradnock G, Morris J, Nunn J, Pine C, Pitt N,Treasure E, White D. Adult dental health survey: Oral health in the United Kingdom 1998. London, The Stationary Office 2000.

This article is the final paper in the adult dental health survey practice series. 